\title{
CT Image Segmentation Method of Liver Tumor Based on Artificial Intelligence Enabled Medical Imaging
}

\author{
Liping Liu $\mathbb{D}^{1},{ }^{1}$ Lin Wang $\mathbb{D}^{1},{ }^{1}$ Dan Xu $\mathbb{D},{ }^{1}$ Hongjie Zhang $\mathbb{D}^{1,2}$ Ashutosh Sharma ${ }^{10},{ }^{3}$ \\ Shailendra Tiwari $\mathbb{D}^{4},{ }^{4}$ Manjit Kaur $\mathbb{D}^{5},{ }^{5}$ Manju Khurana $\mathbb{D},{ }^{6}$ and Mohd Asif Shah $\mathbb{D}^{7}$ \\ ${ }^{1}$ Radiology Department, Affiliated Hospital of Gansu University of Chinese Medicine, Lanzhou, Gansu 73000, China \\ ${ }^{2}$ Affiliated Hospital of Gansu University of Chinese Medicine, Lanzhou, Gansu 73000, China \\ ${ }^{3}$ Institute of Computer Technology and Information Security, Southern Federal University, Rostov-on-Don, Russia \\ ${ }^{4}$ Computer Science and Engineering Department, Thapar Institute of Engineering and Technology, Patiala, India \\ ${ }^{5}$ Computer Science Engineering, School of Engineering and Applied Sciences, Bennett University, Greater Noida 201310, India \\ ${ }^{6}$ Computer Science and Engineering Department, Thapar Institute of Engineering and Technology, Patiala, India \\ ${ }^{7}$ Bakhtar University, Kabul, Afghanistan
}

Correspondence should be addressed to Hongjie Zhang; hongjiezhang1@outlook.com and Mohd Asif Shah; ohaasif@ bakhtar.edu.af

Received 15 March 2021; Revised 5 May 2021; Accepted 17 May 2021; Published 26 May 2021

Academic Editor: Ahmed A. Abd El-Latif

Copyright (c) 2021 Liping Liu et al. This is an open access article distributed under the Creative Commons Attribution License, which permits unrestricted use, distribution, and reproduction in any medium, provided the original work is properly cited.

Artificial intelligence (AI) has made various developments in the image segmentation techniques in the field of medical imaging. This article presents a liver tumor CT image segmentation method based on AI medical imaging-based technology. This study proposed an artificial intelligence-based $K$-means clustering (KMC) algorithm which is further compared with the region growing (RG) method. In this study, 120 patients with liver tumors in the Post Graduate Institute of Medical Education \& Research Hospital, Chandigarh, India, were selected as the research objects, and they were classified according to liver function (Child-Pugh), with 58 cases in grade A and 62 cases in grade B. The experimentation indicates that liver tumor showed low density on plain CT scan, moderate enhancement in the arterial phase of the enhanced scan, and low-density filling defect in the involved blood vessel in the portal venous phase (PVP). It was observed that the CT examination is more sensitive to liver metastasis than hepatocellular carcinoma $(P<0.05)$. The outcomes obtained depict the good deposition effect of lipiodol chemotherapy emulsion (LCTE) in the contrast group with rich blood type accounted for $53.14 \%$ and the patients with the poor blood type accounted for $25.73 \%$ showed poor deposition effect. The comparison with the state-of-the-art method reveals that the segmentation effect of the $\mathrm{KMC}$ algorithm is better than that of the conventional RG method.

\section{Introduction}

The liver is one of the important organs for metabolism in the human internal organs. Because of its participation in the blood transport system, the incidence of liver diseases has been on the rise [1]. Related statistics have proved that 360 million people in the world suffer from abnormal liver dysfunction, and about 1.1 million new hepatocellular carcinoma (HCC) patients are added each year, of which about $25 \%$ are from China and about $20 \%$ are from India [2]. Liver tumor is featured with fast proliferation, high deterioration, easy relapse, and easy metastasis. At present, more than $75 \%$ of patients undergoing liver tumor tissue resection have suffered from the relapse. Overall, the effect of surgical treatment of liver tumors is relatively low $[3,4]$. Therefore, in clinical medicine, there is an urgent need for a diagnosis method to effectively identify the liver tumor lesion area.

In recent years, AI has made further developments in image segmentation for deep learning and machine learning [5-7]. The convolutional neural network (CNN) of deep learning has super recognition and segmentation capabilities and is widely used in image classification and recognition 
[8-10]. AI in the medical field has strong application value in the diagnosis and treatment of diseases [11]. The imaging data of clinical medicine in radiotherapy continues to increase. Conventional processing methods cannot accumulate data, while AI can process massive amounts of data in an orderly manner, perform effective learning based on massive amounts of data, and accumulate knowledge like a doctor $[12,13]$. With the continuous advancement of medical imaging technology, CT takes computer equipment as the core and greatly improves the clinical diagnostic efficacy of various diseases. Due to the rapid inspection of CT images, clear images, and low-cost difference, it is the most common detection method in the diagnosis of liver tumors [14-16].

There are various clinical applications of artificial intelligence reported in the literature for various liver imaging tasks. The tabular representation of these applications is incited in Table 1.

The segmentation of liver structures has great diagnostic importance of determination of vascular diseases using computed tomography (CT) scans. The manual segmentation processes are error prone and time-consuming; therefore, automated segmentation procedures have gained the importance using the applications of deep learning. Dogra et al. [23] utilized the convolutional neural network(CNN-) based approach for liver vasculature segmentation achieving high accuracy rate with Dice coefficient of 0.83 . Lu et al. [24] utilized the CT scans for liver localization and segmentation using the CNN-based approach which achieved high accuracy and efficiency value. A seven-layered CNN model was prepared by $\mathrm{Li}$ et al. [25] for the segmentation of CT images using a tumor segmentation method which achieved a precision rate of $82.67 \%$. A multichanneled CNN-based method was presented by the authors which achieved high robustness as well as accuracy for the detection of liver tumor segments using the CNN network [26]. The performance of artificial intelligencebased methods is exceptional when it comes to liver segmentation approaches. The automated segmentation methods have shown the great potential using deep learning in order to enable the clinical practices for the achievement of more accurate medical treatment [27-35].

The diagnostic relevance of image segmentation for liver tumor detection laid the foundation for this research. This article contributes by proposing a liver tumor CT image segmentation method based on artificial intelligence (AI) medical imaging equipment. The study utilizes 120 patients with liver tumors in the Post Graduate Institute of Medical Education \& Research Hospital, Chandigarh, India, as the research objects, and they were classified according to liver function (Child-Pugh), with 58 cases in grade A and 62 cases in grade $\mathrm{B}$. An artificial intelligence-based $K$-means clustering (KMC) algorithm is proposed in this work which indicates that the liver tumors are low density on the plain CT scan. The novelty of this work lies in moderate enhancement in the arterial phase of the enhanced scan and low-density filling defect in the involved blood vessel in the portal venous phase (PVP) using the proposed approach. The KMC algorithm based on the AI medical imaging equipment was compared with the performance of the RG method in the reconstruction model and applied to the segmentation of CT images of 120 patients with liver tumors. It was observed that the CT examination is more sensitive to liver metastasis than hepatocellular carcinoma, and it was revealed that the segmentation effect of the KMC algorithm is better than that of the conventional RG method.

The rest of this article is arranged as follows: Section 2 presents the materials and methods followed by the experimental results and analysis in Section 3. Section 4 presents the discussion of the article trailed by the concluding remarks in Section 5.

\section{Materials and Methods}

This section presents the general dataset information of the article, the diagnostic criteria, and the treatment method involved. CT examination of patients with liver tumors along with the proposed KMC algorithm is explained in this section of the article.

2.1. Dataset Information. 120 patients who were medically diagnosed with liver tumors in the Post Graduate Institute of Medical Education \& Research Hospital, Chandigarh, India, from November 2018 to October 2019 were selected as the research objects, of which 67 were males and 53 were females. They were graded according to Child-Pugh, with 58 cases in grade A and 62 cases in grade B. The study had been approved by the Medical Ethics Committee of Post Graduate Institute of Medical Education \& Research Hospital.

The inclusion criteria were defined as follows: patients with liver tumor diagnosed by clinical CT imaging; patients with complete imaging data; and, patients aged $20-75$ years old.

The exclusion criteria were defined as follows: patients clinically diagnosed as liver tumor but without direct evidence; patients without HCC resection; patients with major medical diseases; and, patients with unclear CT images.

2.2. Diagnosis Criterion and Treatment Methods. The clinical diagnostic criteria of HCC could be determined as follows: (1) alpha-fetoprotein $>400 \mu \mathrm{g} / \mathrm{L}$ : the metastatic HCC, pregnant embryonic tumors, and active liver disease could be excluded; lump could be found in the liver; the spaceoccupying lesion (SOL) of HCC characteristics was determined by CT imaging; (2) alpha-fetoprotein $\leq 400 \mu \mathrm{g} / \mathrm{L}$ : the SOL of HCC characteristics could be determined by two imaging examinations; two HCC markers were found to be positive; (3) the clinical manifestations of HCC were found, and the lesion had metastasized to the outside of the liver.

Observation indicators of hepatic angiography: liver tumors are divided into two types according to their arterial blood supply and actual staining conditions: one is bloodrich, which means that the blood supply arteries are significantly thickened, blood flow is disordered, and the arterial phase can be observed in many parts of the blood sinusoid abnormal imaging situation, and the liver tumor cells in the parenchymal stage stain very significantly. One is blood-poor, that is, there is no significant thickening of the 
TABle 1: Artificial intelligence practices in clinical investigation of liver diseases.

\begin{tabular}{|c|c|c|c|c|}
\hline $\begin{array}{l}\text { Sr. } \\
\text { no. }\end{array}$ & Technique & Task & Performance metrics & Reference \\
\hline 1 & Deep learning method & Detection of fatty liver anomalies & $\begin{array}{l}\text { Accuracy, sensitivity, and } \\
\text { specificity }\end{array}$ & {$[17]$} \\
\hline 2 & Deep learning method & $\begin{array}{l}\text { Differentiation and detection of various } \\
\text { focal liver lesions }\end{array}$ & $\begin{array}{l}\text { Accuracy, sensitivity, and } \\
\text { specificity }\end{array}$ & {$[18]$} \\
\hline 3 & Deep learning method & Estimation of liver steatosis & $\begin{array}{l}\text { Accuracy, sensitivity, and } \\
\text { specificity }\end{array}$ & {$[19]$} \\
\hline 4 & $\begin{array}{c}\text { Shear wave elastography-based machine } \\
\text { learning method }\end{array}$ & Classification of chronical liver diseases & $\begin{array}{l}\text { Accuracy, sensitivity, and } \\
\text { specificity }\end{array}$ & {$[20]$} \\
\hline 5 & $\begin{array}{c}\text { Deep correlation-based analysis using } \\
\text { multiple kernel learning }\end{array}$ & Liver tumor discrimination & $\begin{array}{l}\text { Accuracy, sensitivity, and } \\
\text { specificity }\end{array}$ & {$[21]$} \\
\hline 6 & $\begin{array}{c}\text { Magnetic resonance imaging-based machine } \\
\text { learning }\end{array}$ & $\begin{array}{l}\text { Treatment response prediction for } \\
\text { chronical liver diseases }\end{array}$ & $\begin{array}{l}\text { Accuracy, sensitivity, and } \\
\text { specificity }\end{array}$ & {$[22]$} \\
\hline
\end{tabular}

blood supply arteries, no abnormal sinus angiography is observed in the arterial phase, and liver tumor cells in the parenchymal phase are relatively lightly stained.

2.3. CT Examination of Patients with Liver Tumors. The GE light Speed VCT 64-slice spiral CT scanner was used to instruct patients to pay attention to fasting. Before scanning, patients were instructed to remove metal jewelry on the chest, to perform an iodine allergy test in advance and to explain the scanning process, so that the patient was in a supine position and kept calm and breathe smoothly. The plain scan was performed from left to right firstly and then from top to bottom, and the scan range was from the carina to the fifth lumbar vertebra. After the plain scan was complete, the enhanced scan could be carried out. The scanning layer thickness was $0.625 \mathrm{~mm}$, the scanning interval was $0.5 \mathrm{~mm}$, the pitch was $0.984: 1$, the matrix was $512 \times 512$, the voltage was $120 \mathrm{kV}$, and the current was $220-500 \mathrm{~mA}$.

The contrast agent was injected using the bolus injection method. Firstly, $15 \mathrm{~mL}$ of saline was injected from the vein to check for subcutaneous leakage. The injection rate of contrast agent iohexol injection was $3.5 \mathrm{~mL} / \mathrm{s}$, and the injection volume was $70-80 \mathrm{~mL}$. $35 \mathrm{~mL}$ of saline was injected with the same way to reduce the concentration of the contrast agent in the superior vena cava. After the contrast was injected completely, the arterial phase was scanned for 25 seconds, PVP was scanned for 50 seconds, and the delay phase was scanned for 150 seconds.

2.4. Proposed KMC Algorithm. This article used the iterative approach of $K$-mean clustering (KMC) that generally partitions the data into different non-overlapping subclusters using certain objective functions. The input data undergoes certain pretreatment which is further followed by the setting of initial parameters and objective function to form the clusters. The flow chart of the KMC algorithm is given in Figure 1.

The original image from the data collected is input to the system for pretreatment involving various image preprocessing methods for removing the variability in the images. Then, the initial parameters are selected for the KMC algorithm defining an objective function. The cluster centers

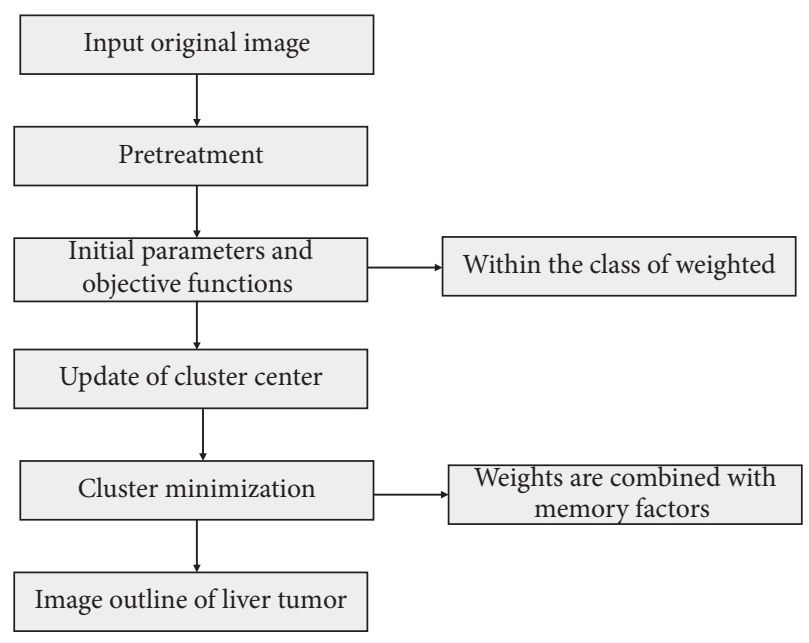

FIgURE 1: The flow chart of the KMC algorithm.

are updated for obtaining the cluster minimization, and then, the segmentation outline is obtained using the KMC algorithm for the liver tumor. The KMC algorithm follows the sequential steps:

(i) Knumber of clusters are specified which are selected randomly

(ii) Parameter initialization is done using the centroid data points for the specification of the objective function

(iii) Iteratively, the cluster centers are updated

(iv) Memory factor is combined with the weight vectors to minimize the number of clusters

(v) Outline of the tumor portion is obtained using these minimized cluster points

The KMC algorithm was a clustering method in image segmentation, which mainly was to distinguish the cluster from the smallest clustering error. It was assumed that the set $A=\left\{a_{1}, a_{2}, \ldots, a_{M}\right\}, a_{n} \in R^{c},(m=1,2, \ldots, M)$; the KMC algorithm could divide the set $A$ into $M$ clusters according to the clustering criterion, namely, $B_{1}, B_{2}, \ldots, B_{M}$; then, the objective function of the KMC algorithm could be expressed as follows: 


$$
K\left(p_{1}, p_{2}, \ldots, p_{M}\right)=\sum_{i-1}^{M} \sum_{k-1}^{N} J\left(a_{i} \in B_{l}\right)\left\|a_{i}-n_{l}\right\|^{2}
$$

where $J$ is the constant and $n$ is the total number of clusters, and in equation (1), $\sum_{i-1}^{M} J\left(a_{i} \in B_{l}\right)\left\|a_{i}-n_{l}\right\|^{2}$ represented the intraclass variance. $K_{\text {sum }}=K\left(p_{1}, p_{2}, \ldots, p_{M}\right)$ was adopted to simplify the equation. The following equation could be applied to calculate the minimum value of the largest clustering error:

$$
K_{\max }=\max _{1 \leq l \leq N} \sum_{i=1}^{M} J\left(a_{i} \in B_{l}\right)\left\|a_{i}-n_{l}\right\|^{2} .
$$

Adopting the above equation was hard for operation, and an objective function was added to obtain the intraclass weight. The specific equation is as follows:

$$
K_{\lambda}=\sum_{l-1}^{N} \lambda_{l}^{q} \sum_{i-1}^{M} J\left(a_{i} \in B_{l}\right)\left\|a_{i}-n_{l}\right\|^{2}, \quad \lambda_{l} \geq 0, \sum_{l-1}^{N} \lambda_{l}^{q}=1, \text { and } 0 \leq \lambda \leq 1,
$$

where $\lambda$ indicates the eigen value that is obtained for $q$ number of clusters.

The weight of each cluster was different, so the cluster had to be minimized. The minimization equation of $\lambda_{l} \geq 0$ is as follows:

$$
\begin{aligned}
& \lambda_{l}=\frac{\mu_{l}^{1 /(1-q)}}{\sum_{l^{\prime}-1}^{N} \mu_{l^{\prime}}^{1 /(1-q)}}, \\
& \mu_{l}=\sum_{i=1}^{M} J\left(z_{i} \in B_{l}\right)\left\|z_{i}-n_{l}\right\|^{2} .
\end{aligned}
$$

It was necessary to combine the weight with the memory factor, so as to enhance the stability of the KMC algorithm:

$$
\lambda_{l}^{(i)}=\theta \lambda_{l}^{(i-1)}+(1-\theta)\left(\frac{\mu_{l}^{1 /(1-q)}}{\sum_{l^{\prime}-1}^{N} \mu_{l^{\prime}}^{1 /(1-q)}}\right), \quad 0 \leq \theta \leq 1 .
$$

In equations (4)-(6), $\mu$ indicates the average value for $q$ number of clusters at an angle $\theta$ ranging from 0 to 1 .

\section{Results}

The result analysis in this section includes the grouping of patients into two different groups based on the number of tumors; CT imaging feature analysis of patients with liver tumor was followed by the liver tumor detection and segmentation results. Diagnostic results are then presented followed by the comparative assessment of the KMC algorithm with the conventional region growing (RG) method.

3.1. Basic Information of Patients in Two Groups. The basic data of the two groups of patients were counted statistically before treatment. The two levels of patients are indicated in Figure 2 (Level A and Level B) as per the number of tumors in patients of two groups. Figure 3 depicts the comparative

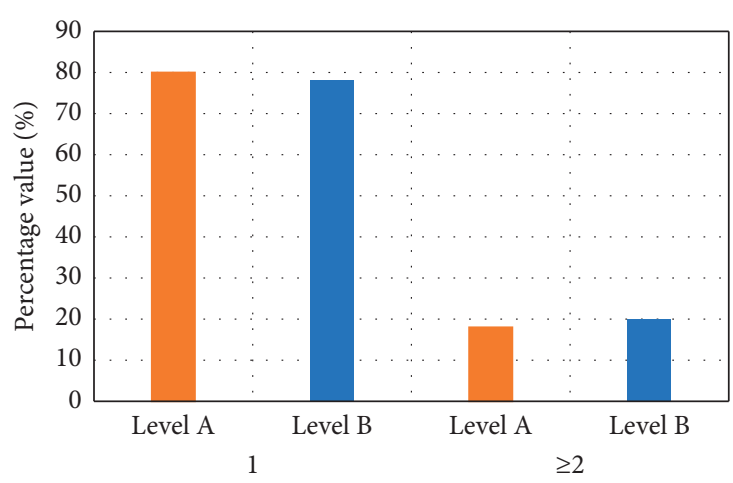

Figure 2: Number of tumors in patients of two groups.

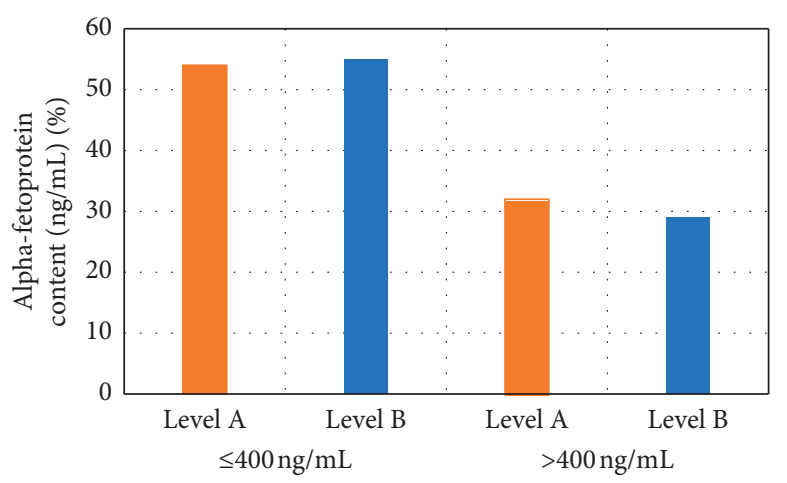

Figure 3: Comparison on alpha-fetoprotein contents of patients.

representation of tumors in patients of two groups in terms of alpha-fetoprotein contents of patients.

There were 67 males and 53 females, with an average age of $52.38 \pm 6.21$ years. Among them, there were 98 patients with single tumors and 37 patients with alpha-fetoprotein content $>400 \mathrm{ng} / \mathrm{mL}$ (as shown in Figure 3); there were 45 patients with tumors larger than $6 \mathrm{~cm}$ in diameter and 71 patients with tumor diameter of smaller than $6 \mathrm{~cm}$. The above differences were not statistically significant $(P>0.05)$.

3.2. CT Imaging Features of Patients with Liver Tumors. Typical CT features of patients with liver tumors are described as follows: plain scan showed mostly low-density or isodensity, enhanced scan showed that the arterial phase was mild to moderate enhanced (nourishing arteries within tumor thrombi could be found), and low-density filling in the blood vessels involved in PVP was not complete (as given in Figure 4). There was no obvious abnormality in the size and shape of the liver, the surface was not smooth, and the density of the posterior segment of the right liver lobe was reduced.

The boundary with the normal liver parenchyma was not clear; the arterial PVP was developed after enhancement, the right lobe of the liver showed irregular enhancement visibly, and the VPV density decreased (as shown in Figure 5). The intrahepatic bile duct and hepatic duct were not dilated. The volume of the gallbladder increased, the wall was still smooth, and double cavities were visible. The common bile 


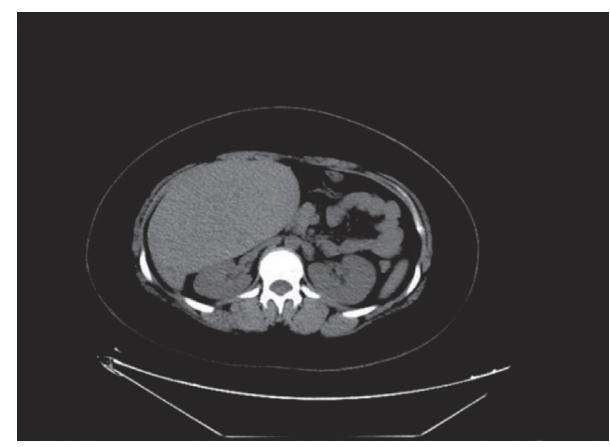

FIgURE 4: Low-density filling defect of the blood vessel in PVP.

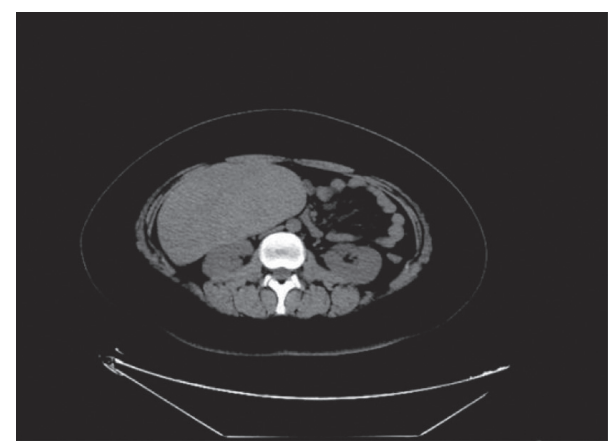

FIGURE 5: Obviously irregular enhancement of the right lobe of the liver.

duct was not dilated. The size and shape of the spleen and pancreas were normal, and there was no abnormal density in the parenchyma. The uncinate process of the head of the pancreas was sharp, and the pancreatic duct was not dilated. The gap could be found among peripancreatic fat tissues. There were no obvious swollen lymph nodes in the abdominal cavity and retroperitoneum.

\subsection{Liver Tumor Detection and Segmentation. After the} analysis of CT imaging features of patients with liver tumor detection, segmentation based on the KMC algorithm is performed whose outcomes are depicted in Figure 6.

This figure represents the original image followed by KMC algorithm-based liver segmentation, and then, tumor segmentation is accomplished using this method. The artificial intelligence-based $K$-means clustering algorithm indicates that the liver tumors are low density using plain CT scan. The moderate enhancement is witnessed in the arterial phase of the enhanced scan and low-density filling defect in the involved blood vessel in the portal venous phase (PVP). The detection and segmentation outcomes are further evaluated in terms of diagnostic evaluation parameters.

3.4. Diagnosis Results on CT Images of Liver Tumor. All 120 patients were examined by CT images. The diagnostic results are analyzed in terms of sensitivity and specificity which are obtained by using the values of true positives (TP), true negatives (TN), false positives (FP), and false negatives (FN).
The formulation of these evaluation parameters are depicted in the following equations:

$$
\begin{aligned}
& \text { sensitivity }=\frac{T P}{T P+F N}, \\
& \text { specificity }=\frac{T N}{T N+F P} .
\end{aligned}
$$

The percentage analysis of sensitivity and specificity of CT examination to the liver tumor for hepatocellular carcinoma (HCC) and liver metastasis cases is indicated in Figure 7.

There were 34 of 64 HCC cases confirmed by pathology that were found by CT; 88 of 103 patients with liver metastases were found by CT; there were 3 cases of hyperplastic nodules, 2 cases of liver abscess, and 5 cases of neuroendocrine tumors. Compared with the sensitivity to HCC, CT examination was more sensitive to liver metastases with great difference $(P<0.05)$.

3.5. Comparison on Reconstruction Performances of Proposed KMC Algorithm with State-of-the-Art Region Growing $(R G)$ Method. The performance of the KMC algorithm based on AI medical imaging equipment in the reconstruction model was compared with that of the region growing (RG) method and applied to the CT image segmentation of 120 patients with liver tumors. The graphical representation of the comparison in terms of Dice coefficient and precision value for both the methods is shown in Figure 8 .

The segmentation effect of the KMC algorithm is better than that of the RG method which shows a better recall rate under a fixed initial point and growth criterion and can accurately segment liver tumor images.

3.6. Grouping Based on CT Examination for Patients with Liver Tumors. 120 patients with liver tumors were divided into blood-rich, blood-poor, and no-blood supply types. Figure 9 reveals that there were 63 blood-rich patients, 30 blood-poor patients, and 27 patients without blood supply, so there was no visible difference $(P>0.05)$.

The type of drug deposition could reflect the richness of the blood supply in patients with liver tumors. Blood-rich patients showed better LCTE deposition effects, while bloodpoor patients and patients with no blood supply showed poor LCTE deposition effects.

\section{Discussion}

Early liver tumors do not have significant clinical features. Generally, it is already in the middle or even advanced stage of cancer when special symptoms appear [36]. For many years, the liver cancer treatment team has communicated with each other through multiple disciplines [37], using CT imaging to detect liver tumors. Eivers et al. (2018) [38] studied the sensitivity and specificity of liver tumors by CT examination and contrast technology; it was found that the degree of malignancy of liver metastases was relatively high, and the postoperative prognosis effect was poor; CT 


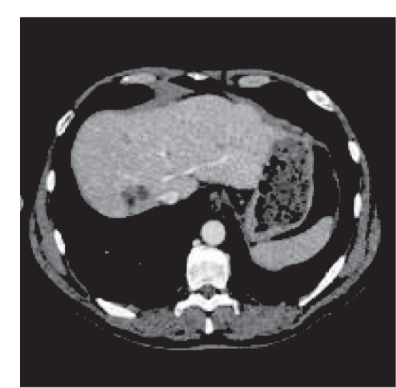

Original input image

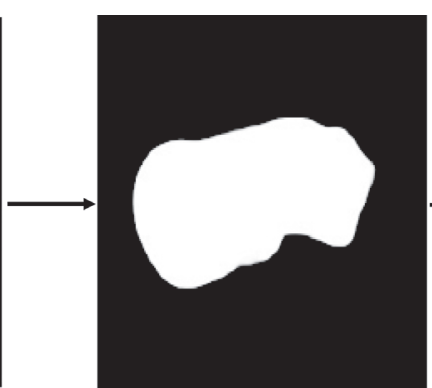

Liver segmentation

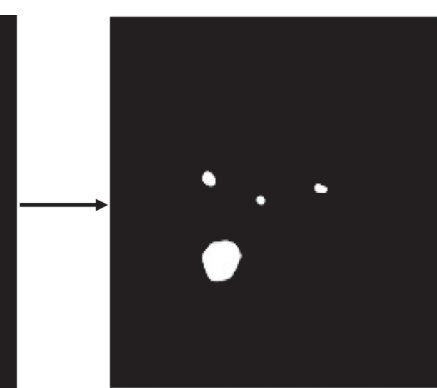

Tumor segmentation

Figure 6: Liver tumor detection and segmentation outcomes.

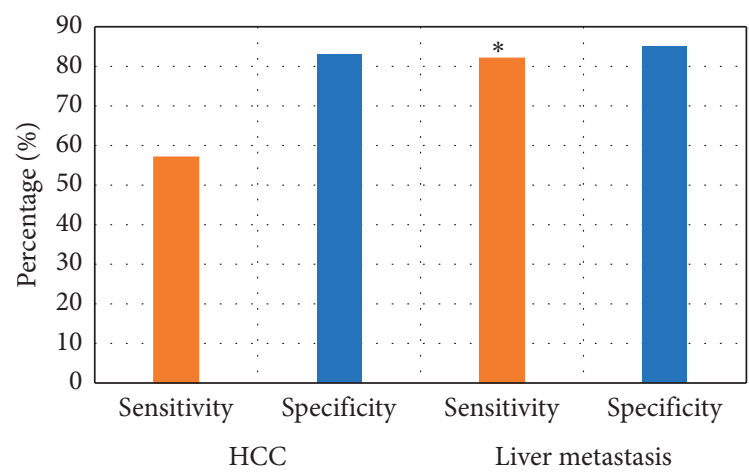

FIgURE 7: Sensitivity and specificity of CT examination to the liver tumor. Note: ${ }^{*}$ indicates that the sensitivity difference was dramatic in contrast to the HCC $(P<0.05)$.

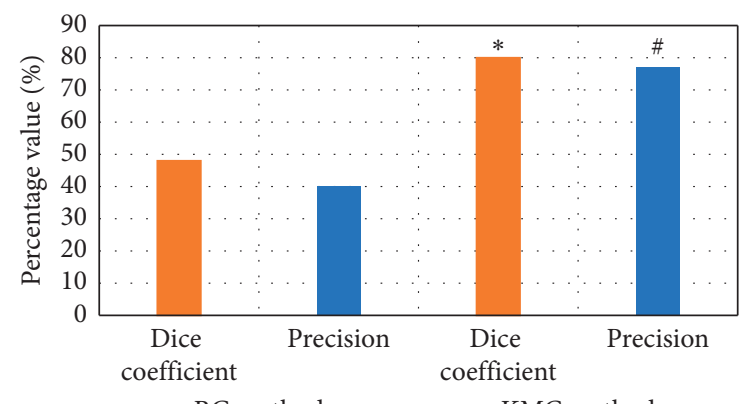

FIgure 8: Comparison on dice coefficient and precision of two algorithms. Note: ${ }^{*}$ indicates that the dice coefficient was obviously different in contrast to the RG method $(P<0.05)$; and, \# suggested that the precision showed great difference in contrast to the $R G$ method $(P<0.05)$.

examination was more sensitive to liver metastases compared with the sensitivity to HCC. It was consistent with the results of this study, indicating that CT examination showed high diagnostic value of liver metastasis.

With the continuous updating of new imaging equipment in clinical medicine, AI has also developed, which provides basic technical support for CT examinations to diagnose cancer [21, 23, 39-42]. Based on AI medical imaging equipment, 120 patients with liver tumors were grouped, blood-rich patients accounted for $53.14 \%$, deposition effect of LCTE was better, blood-poor patients accounted for $25.73 \%$,

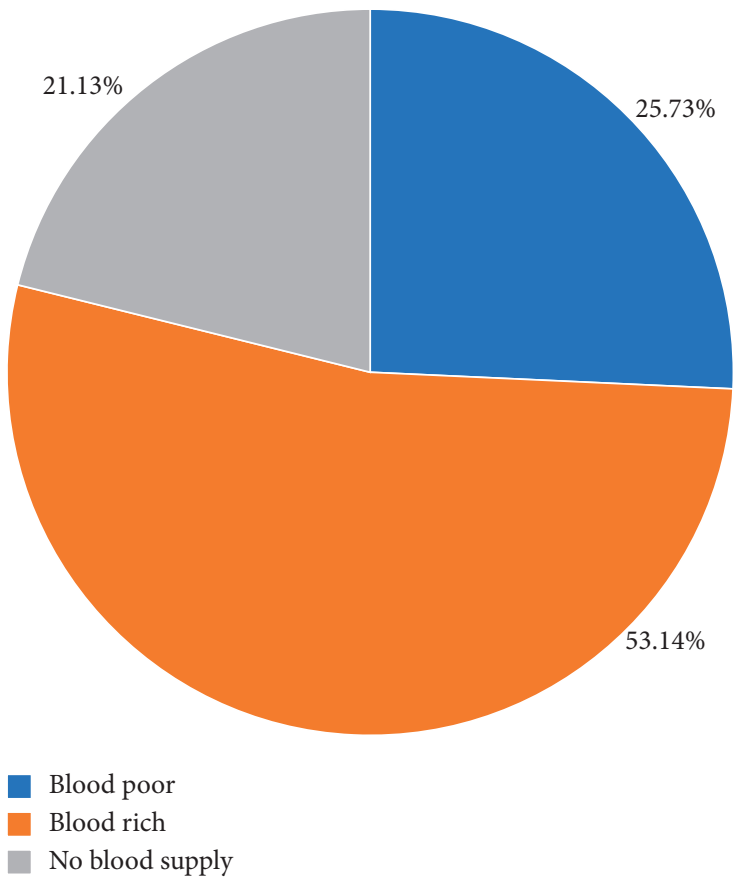

FIGURE 9: Grouping based on CT examination for patients with liver tumors.

and deposition effect of LCTE was poor. The type of drug deposition can reflect the richness of the blood supply arterial phase in patients with liver tumors. The microvessel density of blood-rich liver tumor patients was relatively high, and the LCTE was better tolerated, while blood-poor patients and patients with no-blood supply type tumors had lower microvessel density and lower LCTE that can be accommodated, which was consistent with the research results of Kanona et al. (2017) [43].

\section{Conclusion}

The KMC algorithm based on AI medical imaging equipment was compared with the RG method on performance in the reconstruction model, and it was applied to the CT image segmentation of 120 patients with liver tumors. The segmentation effect of the KMC algorithm was better than that of the RG method, so it could accurately segment the liver tumor images. CT imaging of liver tumors mainly 
manifested as low-density or isodensity on plain scans and light to moderate enhancement in the arterial phase of enhanced scans to enhance low-density filling defects in the blood vessels involved in PVP. CT examination was of high diagnostic value for liver metastases. Blood-rich patients with liver tumors showed relatively high microvessel density and good tolerance for LCTE. The diagnosis results of CT examinations were closely related to the experience level of doctors, and the sample size of this study was small. All in all, CT examination based on AI medical imaging was of high diagnostic value for liver metastases, showing high consistency with pathological test results.

\section{Data Availability}

All data used to support the findings of the study are included within the article.

\section{Conflicts of Interest}

The authors declare that they have no conflicts of interest.

\section{Acknowledgments}

The authors deeply acknowledge the contribution of the Post Graduate Institute of Medical Education \& Research, Chandigarh, India, for providing the CT image data of liver tumor for the study.

\section{References}

[1] C. Cassinotto, A. Denys, F. Gay et al., "Radiofrequency ablation of liver tumors: no difference in the ablation zone volume between cirrhotic and healthy liver," Cardiovascular and Interventional Radiology, vol. 41, no. 6, pp. 905-911, 2018.

[2] Y. Sun, N. Zhang, Y. L. Ding et al., "Effect of lipid metabolism disorder on liver function in patients with malignant tumors after chemotherapy: a case-control study," Lipids in Health and Disease, vol. 18, no. 1, p. 108, 2019.

[3] M. Kaur and D. Singh, "Multiobjective evolutionary optimization techniques based hyperchaotic map and their applications in image encryption," Multidimensional Systems and Signal Processing, vol. 32, no. 1, pp. 281-301, 2021.

[4] C. Bhardwaj, S. Jain, and M. Sood, "Hierarchical severity grade classification of non-proliferative diabetic retinopathy," Journal of Ambient Intelligence and Humanized Computing, vol. 12, no. 2, pp. 2649-2670, 2020.

[5] J. Shindoh, Y. Nishioka, and M. Hashimoto, "Bilateral anatomic resection of the ventral parts of the paramedian sectors of the liver with total caudate lobectomy for deeply/centrally located liver tumors: a new technique maximizing both oncological and surgical safety," Journal of Hepato-BiliaryPancreatic Sciences, vol. 24, no. 12, pp. E10-E16, 2017.

[6] M. Kaur, D. Singh, and V. Kumar, "Color image encryption using minimax differential evolution-based 7D hyper-chaotic map," Applied Physics B, vol. 126, no. 9, pp. 1-19, 2020.

[7] C. Bhardwaj, S. Jain, and M. Sood, "Diabetic retinopathy lesion discriminative diagnostic system for retinal fundus images," Advanced Biomedical Engineering, vol. 9, pp. 71-82, 2020.

[8] R. Stephens, G. Tredwell, K. Knox et al., " $" 99 \mathrm{~m}$ Tc-radiolabeled composites enabling in vivo imaging of arterial dispersal and retention of microspheres in the vascular network of rabbit lungs, liver, and liver tumors," International Journal of Nanomedicine, vol. 14, pp. 889-900, 2019.

[9] S. Almotairi, G. Kareem, M. Aouf, B. Almutairi, and M. A.-M. Salem, "Liver tumor segmentation in CT scans using modified segnet," Sensors, vol. 20, no. 5, p. 1516, 2020.

[10] S. Naeem, A. Ali, S. Qadri et al., "Machine-Learning based hybrid-feature analysis for liver cancer classification using fused (MR and CT) images," Applied Sciences, vol. 10, no. 9, p. 3134, 2020.

[11] D. Li, Z. Cheng, G. Chen et al., "A multimodality imagingcompatible insertion robot with a respiratory motion calibration module designed for ablation of liver tumors: a preclinical study," International Journal of Hyperthermia, vol. 34, no. 8, pp. 1194-1201, 2018.

[12] D. A. Hashimoto, E. Witkowski, L. Gao, O. Meireles, and G. Rosman, "Artificial intelligence in anesthesiology: current techniques, clinical applications, and limitations," Anesthesiology, vol. 132, no. 2, pp. 379-394, 2020.

[13] G. Dhiman, K. K. Singh, M. Soni et al., "MOSOA: a new multiobjective seagull optimization algorithm," Expert Systems with Applications, vol. 167, Article ID 114150, 2020.

[14] C. Leiva-Salinas, B. Jiang, and M. Wintermark, "Computed tomography, computed tomography angiography, and perfusion computed tomography evaluation of acute ischemic stroke," Neuroimaging Clinics of North America, vol. 28, no. 4, pp. 565-572, 2018.

[15] G. Dhiman, D. Oliva, A. Kaur et al., "BEPO: a novel binary emperor penguin optimizer for automatic feature selection," Knowledge-Based Systems, vol. 211, Article ID 106560, 2021.

[16] C. Bhardwaj, S. Jain, and M. Sood, "Diabetic retinopathy detection from eye fundus images with parameter tuning for convolutional neural networks," in Proceedings of the 2019 5th International Conference On Signal Processing, Computing And Control (ISPCC), pp. 13-18, IEEE, Waknaghat Solan, India, October 2019.

[17] M. Biswas, V. Kuppili, D. R. Edla et al., "Symtosis: a liver ultrasound tissue characterization and risk stratification in optimized deep learning paradigm," Computer Methods and Programs in Biomedicine, vol. 155, pp. 165-177, 2018.

[18] T. M. Hassan, M. Elmogy, and E.-S. Sallam, "Diagnosis of focal liver diseases based on deep learning technique for ultrasound images," Arabian Journal for Science and Engineering, vol. 42, no. 8, pp. 3127-3140, 2017.

[19] B. R. Murlidhar, R. K. Sinha, E. T. Mohamad, R. Sonkar, and M. Khorami, "The effects of particle swarm optimisation and genetic algorithm on ANN results in predicting pile bearing capacity," International Journal of Hydromechatronics, vol. 3, no. 1, pp. 69-87, 2020.

[20] A. Sharma, M. D. Ansari, and R. Kumar, "A comparative study of edge detectors in digital image processing," in Proceedings of the 2017 4th International Conference on Signal Processing, Computing and Control (ISPCC), pp. 246-250, IEEE, Solan, India, September 2017.

[21] X. Zhang, R. Wang, A. Sharma, and G. Gopal, "Artificial intelligence in cognitive psychology-influence of literature based on artificial intelligence on children's mental disorders," Aggression and Violent Behavior, Article ID 101590, 2021.

[22] B. Ibragimov, D. Toesca, D. Chang, Y. Yuan, A. Koong, and L. Xing, "Development of deep neural network for individualized hepatobiliary toxicity prediction after liver SBRT," Medical Physics, vol. 45, no. 10, pp. 4763-4774, 2018.

[23] J. Dogra, S. Jain, A. Sharma, R. Kumar, and M. Sood, "Brain tumor detection from MR images employing fuzzy graph cut 
technique," Recent Advances in Computer Science and Communications, vol. 13, no. 3, pp. 362-369, 2020.

[24] F. Lu, F. Wu, P. Hu, Z. Peng, and D. Kong, "Automatic 3D liver location and segmentation via convolutional neural network and graph cut," International Journal of Computer Assisted Radiology and Surgery, vol. 12, no. 2, pp. 171-182, 2017.

[25] W. Li, F. Jia, and Q. Hu, "Automatic segmentation of liver tumor in CT images with deep convolutional neural networks," Journal of Computer and Communications, vol. 03, no. 11, pp. 146-151, 2015.

[26] A. Sharma and R. Kumar, "An optimal routing scheme for critical healthcare HTH services-an IOT perspective," in Proceedings of the 2017 Fourth International Conference on Image Information Processing (ICIIP), pp. 1-5, IEEE, Waknaghat Solan, India, December 2017.

[27] G. Rathee, A. Sharma, R. Kumar, F. Ahmad, and R. Iqbal, “A trust management scheme to secure mobile information centric networks," Computer Communications, vol. 151, pp. 66-75, 2020.

[28] C. Bhardwaj, S. Jain, and M. Sood, "Deep learning based diabetic retinopathy prediction of colored fundus images with parameter tuning," in Advances In Computational Intelligence Techniques, pp. 53-62, Springer, Singapore, 2020.

[29] M. A. Alsmirat, F. Al-Alem, M. Al-Ayyoub, Y. Jararweh, and B. Gupta, "Impact of digital fingerprint image quality on the fingerprint recognition accuracy," Multimedia Tools and Applications, vol. 78, no. 3, pp. 3649-3688, 2019.

[30] S. AlZu'bi, M. Shehab, M. Al-Ayyoub, Y. Jararweh, and B. Gupta, "Parallel implementation for 3d medical volume fuzzy segmentation," Pattern Recognition Letters, vol. 130, pp. 312-318, 2020.

[31] B. B. Gupta and M. Quamara, "An overview of Internet of Things (IoT): architectural aspects, challenges, and protocols," Concurrency and Computation: Practice and Experience, vol. 32, no. 21, Article ID e4946, 2020.

[32] H. Wang, Z. Li, Y. Li, B. B. Gupta, and C. Choi, "Visual saliency guided complex image retrieval," Pattern Recognition Letters, vol. 130, pp. 64-72, 2020.

[33] T. Zhang, A. A. Abd El-Latif, N. Wang, Q. Li, and X. Niu, "A new image segmentation method via fusing NCut eigenvectors maps," in Proceedings of the Fourth International Conference on Digital Image Processing (ICDIP 2012), vol. 8334, April 2012, Article ID 833430.

[34] A. Sedik, M. Hammad, F. E. Abd El-Samie, B. B. Gupta, and A. A. Abd El-Latif, "Efficient deep learning approach for augmented detection of Coronavirus disease," Neural Computing \& Applications, vol. 8, pp. 1-18, 2021.

[35] M. Hammad, A. M. Iliyasu, A. Subasi, E. S. Ho, and A. A. Abd El-Latif, "A multitier deep learning model for arrhythmia detection," IEEE Transactions on Instrumentation and Measurement, vol. 70, pp. 1-9, 2020.

[36] H. Lee, S. Lee, J. G. Cha, T. Baek, and K.-m. Yang, "Postmortem computed tomography and computed tomography angiography: cardiothoracic imaging applications in forensic medicine," Journal of Thoracic Imaging, vol. 34, no. 5, pp. 286-298, 2019.

[37] K. Stawiarski, D. Sorajja, and H. Ramakrishna, "Magnetic resonance and computed tomography imaging in patients with cardiovascular implantable electronic devices: analysis of expert consensus data and implications for the perioperative clinician," Journal of Cardiothoracic and Vascular Anesthesia, vol. 32, no. 6, pp. 2817-2822, 2018.
[38] C. R. Eivers, N. Corzo-Menéndez, S. H. Austwick et al., "Computed tomographic arthrography is a useful adjunct to survey computed tomography and arthroscopic evaluation of the canine shoulder joint," Veterinary Radiology \& Ultrasound, vol. 59, no. 5, pp. 535-544, 2018.

[39] A. Sharma and R. Kumar, "Service-level agreement-energy cooperative quickest ambulance routing for critical healthcare services," Arabian Journal for Science and Engineering, vol. 44, no. 4, pp. 3831-3848, 2019.

[40] C. Printz, "Pediatric computed tomography scans: weighing the risks and benefits: a new study suggests that computed tomography-related radiation may increase the risk of brain tumors, but the conversation, physicians say, is complex-and far from settled," Cancer, vol. 125, no. 2, pp. 171-173, 2019.

[41] R. T. Zweifel, P. DiDonato, A. Hartmann, M. Kramer, and K. H. von Pückler, "Improved computed tomography accuracy with a $1-\mathrm{mm}$ versus 2 - or $3-\mathrm{mm}$ slice thickness for the detection of medial coronoid disease in dogs," Veterinary and Comparative Orthopaedics and Traumatology, vol. 33, no. 01, pp. 045-050, 2020.

[42] G. K. Sodhi, S. Kaur, G. S. Gaba, L. Kansal, A. Sharma, and G. Dhiman, "COVID-19: role of robotics, artificial intelligence, and machine learning during pandemic," Current Medical Imaging, 2021.

[43] H. Kanona, I. Rana, C. Offiah, and N. Patel, "Importance of a dedicated neuroradiologist in reporting high-resolution computed tomography for otosclerosis: a retrospective comparison study of 40 patients," The Journal of Laryngology \& Otology, vol. 131, no. 6, pp. 492-496, 2017. 